\title{
Progression and new onset of macular retinoschisis in myopic choroidal neovascularization eyes after Conbercept therapy: a post-hoc analysis
}

\author{
Yanping Zhou ${ }^{1} \cdot$ Shiqi Yang ${ }^{1}$ Yuanzhi Yuan ${ }^{2} \cdot$ Minlu Song ${ }^{1} \cdot$ Fenglei Kuang $^{3} \cdot$ Kun Liu $^{1,4,5} \cdot$ Feng Zhang $^{6} \cdot$ \\ Fenghua Wang ${ }^{1,4,5,7} \cdot$ Xiaodong Sun ${ }^{1,5,7}$
}

Received: 8 April 2018 / Accepted: 18 June 2019 / Published online: 29 July 2019

(c) The Author(s), under exclusive licence to The Royal College of Ophthalmologists 2019

\begin{abstract}
Objectives The objective of this study is to evaluate the progression and new onset of macular retinoschisis (MRS) in the patients treated with intravitreal Conbercept injections for myopic choroidal neovascularization (mCNV).

Methods Post-hoc analysis of $160 \mathrm{mCNV}$ patients included in SHINY study was performed to evaluate the impact of Conbercept injection on MRS in patients with mCNV undergoing intravitreal Conbercept injections. The patients were 3:1 randomized to the study group (three loading dose and thereafter pro re nata [PRN]) and the control group (3 months' sham injection, then one Conbercept injection at month 4 and thereafter PRN). MRS was assessed with optical coherence tomography by masked graders.

Results At baseline, 28 of 122 eyes in study group and 10 of 38 eyes in control group had MRS. At month 3, two patients showed MRS progression and one patient had new onset MRS in study group. No MRS progression nor new onset MRS was found in the control group. At final visit, the cumulative incidence of MRS was 1.3\% (2/160). Both Spearman's correlation and multiple logistic regression demonstrated no association between the progression and new onset of MRS and intravitreal injection frequency (correlation coefficient $=0.017, P=0.851$ and odds ratio $=0.996, P=0.982$ ). In addition, baseline vitreoretinal adhesion was the most likely potential risk factor resulting in MRS progression (odds ratio $=4.566$, $P=0.027)$. Furthermore, MRS progression was more likely to take place in outer retinal layers.

Conclusions The progression and new onset of MRS was not associated with the frequency of intravitreal Conbercept injections.
\end{abstract}

\section{Introduction}

Pathologic myopia, composed of a series of funduscopic changes such as retinoschisis, macular hole $(\mathrm{MH})$, epiretinal

These authors contributed equally: Yanping Zhou, Shiqi Yang

Fenghua Wang

shretina@sjtu.edu.cn

$\triangle$ Xiaodong Sun

xdsun@sjtu.edu.cn

1 Department of Ophthalmology, Shanghai General Hospital (Shanghai First People's Hospital), Shanghai Jiao Tong University School of Medicine, Shanghai, China

2 Department of Ophthalmology, Zhongshan Hospital, Fudan University, Shanghai, China membrane (ERM) and choroidal neovascularization (CNV), is the second common cause of visual impairment and blindness in China [1-3]. Myopic macular retinoschisis (MRS), defined as the splitting of retina in inner and/or outer retinal layers with hyporeflective cystoid spaces and columnar structures [4-6], is present in one-third of pathologic myopic eyes with posterior staphyloma and the

3 R\&D Center, Chengdu Kanghong Biotech Ltd., Sichuan, China

4 Shanghai Jiao Tong University Eye Institute Reading Center, Shanghai, China

5 Shanghai Key Laboratory of Fundus Diseases, 100 Haining Road, Shanghai, China

6 Department of Ophthalmology, Beijing Tongren Hospital, Capital Medical University, Peking, China

7 Shanghai Engineering Center for Visual Science and Photomedicine, Shanghai, China 
MRS prevalence in these eyes ranges from $9 \%$ to $34 \%$ [7-9]. It is not rare that MRS co-existed with myopic CNV (mCNV) [10].

mCNV develops between $5.2 \%$ and $11.3 \%$ of patients with pathologic myopia [3]. Intravitreal injection of antivascular endothelial growth factor (anti-VEGF) agents is the first-line treatment for mCNV [11-13]. However, several case report studies considered that some rare complications such as MH development and new onset MRS may result from anti-VEGF therapy [14-16].

Previous researches demonstrated that the occurrence and progression of MRS may correlate with vitreoretinal interface disturbance $[6,17,18]$. The procedure of intravitreal injection may lead to disturbance of vitreous body and the drug fluid may also aggravate vitreous liquefaction, which will form an inward force. In addition, anti-VEGF therapy could shrink the CNV lesion and lessen the macular oedema [11, 12], which will form an outward force. However, it is unknown whether these two opposite mechanical stretching forces may associate with the new onset MRS or aggravation of MRS. Thus, this study assessed whether there was a correlation between intravitreal injections and the progression and new onset of MRS.

\section{Materials and methods}

This was a post-hoc analysis from SHINY study (www. clinicaltrials.gov, NCT01809223), a randomized, doubleblind, multicentre, sham-controlled study of the safety and efficacy of Conbercept in patients with $\mathrm{mCNV}$ for 9 months' follow-up. The study protocol was approved by the Institution's Ethical Review Commission. The tenets of the Declaration of Helsinki were followed.

The key inclusion criteria of SHINY study were as follows: (1) eyes with spherical equivalent refractive errors of -6.0 dioptres (D) or more and axial length $\geq 26.5 \mathrm{~mm}$; (2) diagnosis of active subfoveal, juxafoveal, or extrafoveal CNV secondary to high myopia; (3) visual impairment due to CNV secondary to high myopia; (4) best-corrected visual acuity (BCVA) score $\geq 24$ and $\leq 73$ ETDRS letters at a starting distance of $4 \mathrm{~m}$. The major exclusion criteria included the following: (1) eyes that were treated with any anti-VEGF agents/corticosteroids/photodynamic therapy within 6 months and/or that underwent any intraocular surgery; (2) fibrosis or atrophy involving the centre of fovea in the study eye; (3) combined vitreoretinal diseases, such as current vitreous haemorrhage, ERM that may affect central visual acuity, active infectious ocular inflammation, dense cataract, advanced glaucoma or amblyopia.

Patients in SHINY study were randomized (3:1) to study arm and control arm. Patients in the study arm received Conbercept injections at a dose of $0.5 \mathrm{mg}$ per eye, once a month for first 3 months and thereafter as needed based on the monthly assessment results. Patients in the control arm received sham injection monthly for first 3 months, then $0.5 \mathrm{mg}$ Conbercept per eye at month 4 and thereafter as needed based on the monthly assessment results.

In the present study, two graders from Shanghai Jiao Tong University Eye Institute Reading Center (SJTURC) analysed the spectral domain optical coherence tomography (OCT) imaging independently. The presence of MRS and vitreoretinal adhesion on OCT scans were recorded at baseline, month 3, month 6 and final visit. Vitreoretinal adhesion was referred to a visible high reflection at the interface between vitreous and retina without traction (Figure 1). Inner schisis is defined as the splitting within the retinal nerve fibre layer, ganglion cell layer and the inner plexiform $[5,10]$. Outer schisis is defined as the splitting within the outer plexiform layer and the outer nuclear layer $[4,6]$. Divergences were final approved by chief grader from SJTURC.

Here, MRS was evaluated and classified as 'progression' and 'new onset'. 'Progression' was defined as development of an inner lamellar $\mathrm{MH}$, foveal retinal detachment or fullthickness $\mathrm{MH}$, or an increase of more than $100 \mu \mathrm{m}$ in the height of retinoschisis and an enlargement of the retinoschisis towards new area [6]. Figure 1 showed the progression of MRS after intravitreal injection. The "without progression and new onset of MRS' was defined as without any of the sign of MRS progression mentioned above or without MRS.

In this study, all the data were analysed by using a statistical software programme (SPSS 18.0; SPSS, Inc., Chicago, IL, USA). Continuous variables were compared by independent-samples Student's $t$-tests or paired $t$-tests. Categorical variables were assessed by using Pearson's $\chi^{2}$ tests or Fisher's exact test. Spearman's correlation was performed to detect the potential relationship between clinical information and MRS progression or new onset. Binary logistic regression analysis models were performed to determine the risk factors of MRS progression and new onset. $P<0.05$ was considered to indicate statistical significance in all tests.

\section{Results}

There were 176 patients with active mCNV included in SHINY study. In the present study, 16 patients were excluded for less than 9 months of follow-up or for lack of high quality of OCT imaging for MRS evaluation. Therefore, a total of 160 eyes from 160 patients were included in our study. Among them, 122 eyes got first three Conbercept injections and 38 eyes received first three sham injections. After three loading doses in the study group, two eyes 


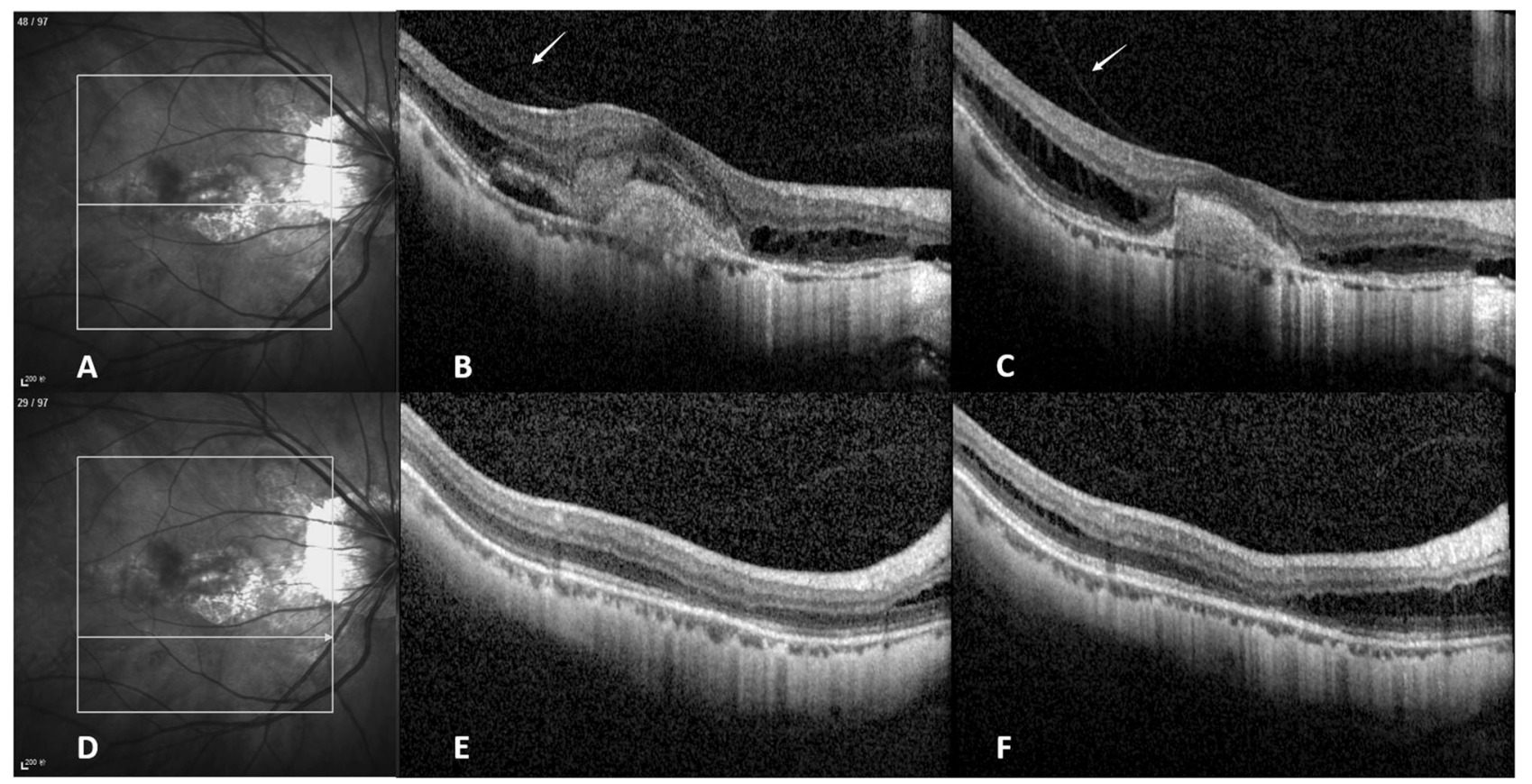

Fig. 1 Representative case of MRS progression. a and $\mathbf{d}$ indicated the scanning position of macula. $\mathbf{b}$ and $\mathbf{e}$ demonstrated the baseline image of optical coherence tomography in myopic choroidal neovascularization eyes coexisted with retinoschisis. $\mathbf{c}$ and $\mathbf{f}$ showed an enlargement of macular retinoschisis toward new area at final visit. Vitreoretinal adhesion was referred to a visible high reflection at the interface between vitreous and retina without obvious traction (white arrows)
Table 1 Comparison of $\mathrm{mCNV}$ eyes with and without the progression and new onset of MRS

\begin{tabular}{llll}
\hline & $\begin{array}{l}\text { With the progression } \\
\text { and new onset of MRS }\end{array}$ & $\begin{array}{l}\text { Without the progression } \\
\text { and new onset of MRS }\end{array}$ & $P$-value \\
\hline Eyes, $n$ & 12 & 110 & 0.313 \\
Age, years & $46 \pm 11$ & $50 \pm 12$ & 0.182 \\
Gender (male/female) & $6 / 6$ & $31 / 79$ & 0.770 \\
Axial length, mm & $28.76 \pm 1.18$ & $28.89 \pm 1.51$ & 0.783 \\
Total injections & $5.1 \pm 1.9$ & $4.9 \pm 1.7$ & 0.766 \\
BCVA improvement, letters & $12.7 \pm 8.5$ & $13.6 \pm 10.9$ & 0.776 \\
CRT decrease, $\mu$ m & $98.9 \pm 104.6$ & $88.9 \pm 116.9$ & $0.016^{*}$ \\
Vitreoretinal adhesion $(\%)$ & $6(50.0)$ & $19(17.3)$ & \\
\hline
\end{tabular}

$B C V A$ best-corrected visual acuity, $C R T$ central retinal thickness, $m C N V$ myopic choroidal neovascularization, $M R S$ macular retinoschisis. ${ }^{*} P$-value $<0.05$
(1.6\%) had MRS progression and one eye $(0.8 \%)$ had new onset MRS. However, no MRS progression nor newonset MRS was found in the control group. The cumulative incidence of MRS was $1.3 \%$ (2/160) after 9 months of follow-up in the present study.

After 9 months of follow-up, a total of 10 eyes got MRS progression and 2 eyes had new-onset MRS in the study group. Potential risk factors for the progression and new onset of MRS were explored in the study group (Table 1). There was no statistical difference in age, gender, axial length, total injections of Conbercept, BCVA improvement and central retinal thickness (CRT) decrease between mCNV eyes with and without the progression and new onset of MRS (all $P>0.05$ ). However, the eyes with the progression and new onset of MRS had greater proportion of vitreoretinal adhesion than the eyes without the progression and new onset of MRS (50.0\%, 6/12 vs. 17.3\%, 19/110, Fisher's exact test, $P=0.016$ ).

Spearman's correlation demonstrated a significant positive correlation between vitreoretinal adhesion and the progression and new onset of MRS $(P=0.007)$, whereas the intravitreal injection frequency was not statistically associated with the progression and new onset of MRS $(P=0.851)$ (Table 2).

Furthermore, multiple logistic regression was performed to determine the risk factors of progression and new onset of MRS in Table 3. After putting age, axial length, total number of intravitreal injections and baseline vitreoretinal 
Table 2 Spearman's correlation between clinical information and the progression and new onset of MRS

\begin{tabular}{lcc}
\hline Various factors & Correlation coefficient & $P$-value \\
\hline Age & -0.105 & 0.249 \\
Axial length & -0.005 & 0.956 \\
Vitreoretinal adhesion & 0.241 & $0.007^{*}$ \\
No. of intravitreal injections & 0.017 & 0.851 \\
\hline
\end{tabular}

MRS macular retinoschisis. $* P$-value $<0.05$

Table 3 Risk factors associated with the progression and new onset of MRS after intravitreal Conbercept injection for co-existed mCNV

\begin{tabular}{llll}
\hline Risk factors & OR & $95 \%$ CI & $P$-value \\
\hline Age & 1.007 & $0.952,1.065$ & 0.806 \\
Axial length & 1.079 & $0.711,1.637$ & 0.722 \\
No. of intravitreal injections & 0.996 & $0.670,1.439$ & 0.982 \\
Vitreoretinal adhesion & 4.566 & $1.189,17.544$ & $0.027 *$ \\
\hline
\end{tabular}

$C I$ confidence interval, $m C N V$ myopic choroidal neovascularization, $M R S$ macular retinoschisis, $O R$ odds ratio. ${ }^{*} P$-value $<0.05$

adhesion into the model, baseline vitreoretinal adhesion was independently and strongly associated with the progression and new onset of MRS (odds ratio $(\mathrm{OR})=4.566, P=$ 0.027). However, the intravitreal injection frequency (total number of intravitreal injections) was shown no effect on it $(\mathrm{OR}=0.996, P=0.982)$.

Detailed characteristics of eyes got MRS progression and new onset (12 eyes from the study arm and 1 eye from the control arm) was summarized in Table 4. There were seven men (54\%) and six women (46\%). Overall, the mean age was $48 \pm 12$ years (range 18-67 years). The mean axial length was $28.7 \pm 1.1 \mathrm{~mm}$ (range 26.6-30.4 mm). Eleven eyes (84.6\%) had baseline MRS. The majority of $69.2 \%$ (9/13) eyes with MRS progression had schisis in the outer retinal layers. Three of 13 eyes (23.1\%) had schisis in both the outer and inner retinal layers. Only one eye had schisis in the inner retinal layers. Moreover, 12 of 13 eyes (92.3\%) had outer schisis progression. In the present study, $84.6 \%$ of MRS (11/13) only involved the outer ETDRS subfield. Baseline vitreoretinal adhesion was present in 7 of 13 eyes. Nine cases of MRS progression were characterized as an enlargement of MRS at outer ETDRS subfields.

\section{Discussion}

In this study, we performed a post-hoc analysis to evaluate the progression and new onset of MRS after intravitreal injection of Conbercept in mCNV eyes. We found that the progression and new onset of MRS was not associated with the number of intravitreal Conbercept injections. The most likely potential risk factor resulting in MRS progression and new onset in those eyes was baseline vitreoretinal adhesion. Furthermore, MRS progression was more likely to occur in outer retinal layers.

The baseline prevalence of MRS was $23.8 \%$ (38/160) in the present study, which is relatively higher than reported prevalence from $6 \%$ to $14.65 \%$ [8, 10]. Such discrepancy might partly due to the different sampling method. In the present study, we included only $\mathrm{mCNV}$ eyes rather than both eyes, thus yielded a higher MRS prevalence in comparison with otherwise normal high myopias.

In the present study, all eyes had marked improvement of visual acuity and a significant decrease of mean CRT after Conbercept therapy for active $\mathrm{mCNV}$ in comparison with baseline. The 9-month cumulative incidence of MRS was $1.3 \%(2 / 160)$ after intravitreal injection of Conbercept, similar to the incidence of $1.7 \%$ reported by Ceklic et al. [10] in mCNV eyes. A total of 10 eyes got MRS progression and 2 eyes had new onset MRS in the study group at final visit. Taken together, the benefit of intravitreal Conbercept therapy for $\mathrm{mCNV}$ outweighs the potential risk of it.

Previous studies suggested that new onset MRS may have been related to the intravitreal injection of anti-VEGF drugs $[10,16]$. However, in the present study, multiple logistic regression analysis indicated that the final total number of intravitreal Conbercept injection was not an independent risk factor for the progression and new onset of MRS (OR $=996, P=0.982)$. To further investigate the potential relationship between MRS development and intravitreal Conbercept injection, we graded the OCT imaging in this study.

Interestingly, baseline vitreoretinal adhesion rate was relatively higher in patients with the progression and new onset of MRS than in patients without the progression and new onset of MRS $(P=0.016)$. The specific mechanism underlying the pathogenesis of retinoschisis remains unknown. Previous studies considered that vitreoretinal interface disturbance [6, 17-19], posterior staphyloma $[20,21]$ and elongation of the axial length [22] were risk factors of retinoschisis formation. Pars plana vitrectomy has been reported to provide a stable condition in patients with myopic MRS [23]. On the other hand, MRS may progress because of persistent vitreoretinal tractions [24].

In patients with age-related macular degeneration, intravitreal injections may induce partial posterior vitreous detachment (PVD) in eyes with baseline focal vitreoretinal adhesion [25]. The rapid impulsion of drug may lead to vitreous perturbation. Compared with non-myopic eyes, synchysis and PVD are rather prevalent in myopic eyes $[26,27]$. The intravitreal injection procedure may lead to disturbance of vitreous body and the drug fluid may exacerbate vitreous liquefaction, which will form an inward force. In addition, the drug fluid mixed with vitreous body potentially breaks the primary density of vitreous body, 


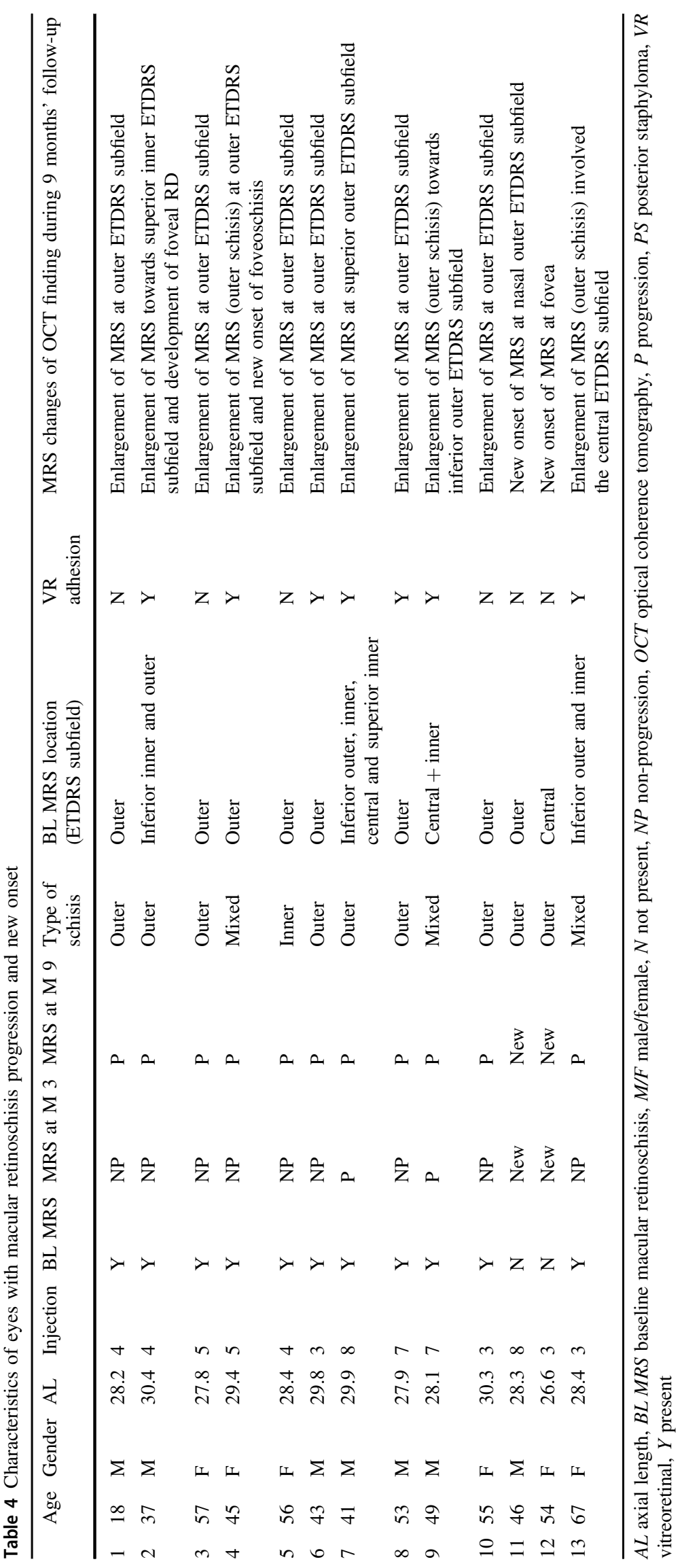


which further deteriorates vitreous complex. This vitreous perturbation consistently inflicts a circumferential force to interact the premacular structure like vitreous adhesion. Thus, the procedure of intravitreal injection should perform in a slow speed to avoid a rapid influx of drug fluid. However, there were six eyes that developed MRS progression or new onset without the presence of baseline vitreoretinal adhesion. To further discuss the potential mechanism of MRS progression or new onset after intravitreal injection of Conbercept, we analysed the characteristics of schisis in our study.

Detailed characteristics showed that the majority of the eyes with MRS progression had schisis in the outer retinal layers (9/13 eyes [69.2\%]). Three of 13 eyes $(23.1 \%)$ had schisis in both outer and inner retinal layers. Only one eye had schisis in the inner retinal layers. Moreover, 12 of 13 eyes $(92.3 \%)$ had outer schisis progression. One explanation is that after Conbercept therapy, the CNV complex shrinks and the ameliorated macular oedema subsequently forming a centripetal retraction of the lesion from the surrounding retinal pigmental epithelium (RPE) monolayer, which induce an outward force. Subfoveal and parafoveal choroidal thickness decreased in eyes with mCNV after anti-VEGF therapy [28]. This may also suggest an outward force after Conbercept therapy. In addition, choroidal vessel contraction after Conbercept therapy may be another explanation. CNV regression and choroidal permeability reduction result from the decrease of VEGF concentrations after Conbercept therapy may be plausible explanations for the outer type schisis progression. In the present study, most MRS progression were involved in outer ETDRS subfield because of the high prevalence of MRS in outer ETDRS subfield and the lobular blood flow of choroid. Taken together, the two opposite forces may be the most probable reason for MRS progression after Conbercept therapy.

As a post-hoc study, there are some limitations. First, the strict inclusion and exclusion criteria prevent us from identifying all independent risk factors associated with MRS progression. Second, posterior staphyloma was rarely recorded in the primary RCT study. Sun et al. [29] suggested that posterior scleral expansion, progressively inducing an outward expansion of choroid, may be the initial pathological trigger of MRS in pathologic myopic eyes. It should be noted that few evidences indicate the disturbance of the scleral collagen array or posterior scleral expansion after anti-VEGF treatment. Thus, a further prospective research is of paramount importance. Third, the follow-up period was not sufficiently to track the MRS development in the present study. Last, being lack of axial length of fellow eyes, the comparison of MRS development between eyes with or without Conbercept injection was unavailable. Therefore, further prospective longitudinal observations are necessary to evaluate the course of MRS development in mCNV eyes.

In conclusion, MRS is not rare in eyes with mCNV. The progression and new onset of MRS may not be correlated to the frequency of intravitreal Conbercept injections. Baseline vitreoretinal adhesion is the most likely potential risk factors resulting in MRS progression. Moreover, MRS progression were more likely to take place in outer retinal layers. Therefore, periodic macular OCT scanning is necessary when $\mathrm{mCNV}$ eyes treated with anti-VEGF therapy.

\section{Summary}

\section{What was known before}

- Macular retinoschisis is not rare in myopic choroidal neovascularization eyes, but whether macular retinoschisis progression is correlated with intravitreal injection of Conbercept remains unknown.

\section{What this study adds}

- Macular retinoschisis progression was not associated with the frequency of intravitreal Conbercept injections.

- Baseline vitreoretinal adhesion is the most likely potential risk factor resulting in macular retinoschisis progression.

Acknowledgements Statistics analysis were supported by Jingye Wang, MD, MPH, University of Hawaii Cancer Center, Honolulu, HI 96813 , USA.

Funding This study was supported by the National Natural Science Foundation of China (81470640 and 81730026), Translational Medicine Grant of Shanghai Jiao Tong University School of Medicine (15ZH4005), Frontier Project of Hospital Development Center (SHDC12016105) and Shanghai Engineering Technology Research Center Construction Project (16dz2251500).

\section{Compliance with ethical standards}

Conflict of interest The authors declare that they have no conflict of interest.

Publisher's note: Springer Nature remains neutral with regard to jurisdictional claims in published maps and institutional affiliations.

\section{References}

1. Xu L, Wang Y, Li Y, Wang Y, Cui T, Li J, et al. Causes of blindness and visual impairment in urban and rural areas in Beijing: the Beijing Eye Study. Ophthalmology. 2006;113:1134 e1131-11. 
2. Xu L, Li J, Cui T, Hu A, Fan G, Zhang R, et al. Refractive error in urban and rural adult Chinese in Beijing. Ophthalmology. 2005;112:1676-83.

3. Wong TY, Ferreira A, Hughes R, Carter G, Mitchell P. Epidemiology and disease burden of pathologic myopia and myopic choroidal neovascularization: an evidence-based systematic review. Am J Ophthalmol. 2014;157:9-25.

4. Fujimoto M, Hangai M, Suda K, Yoshimura N. Features associated with foveal retinal detachment in myopic macular retinoschisis. Am J Ophthalmol. 2010;150:863-70.

5. Sayanagi K, Ikuno Y, Tano Y. Tractional internal limiting membrane detachment in highly myopic eyes. Am J Ophthalmol. 2006;142:850-2.

6. Shimada N, Tanaka Y, Tokoro T, Ohno-Matsui K. Natural course of myopic traction maculopathy and factors associated with progression or resolution. Am J Ophthalmol. 2013;156:948-57.

7. Baba T, Ohno-Matsui K, Futagami S, Yoshida T, Yasuzumi K, Kojima A, et al. Prevalence and characteristics of foveal retinal detachment without macular hole in high myopia. Am J Ophthalmol. 2003;135:338-42.

8. Henaine-Berra A, Zand-Hadas IM, Fromow-Guerra J, GarciaAguirre G. Prevalence of macular anatomic abnormalities in high myopia. Ophthalmic Surg Lasers Imaging Retin. 2013;44:140-4.

9. Takano M, Kishi S. Foveal retinoschisis and retinal detachment in severely myopic eyes with posterior staphyloma. Am J Ophthalmol. 1999;128:472-6.

10. Ceklic L, Munk MR, Wolf-Schnurrbusch U, Gekkieva M, Wolf S. Visual acuity outcomes of Ranibizumab treatment in pathologic myopic eyes with macular retinoschisis and choroidal neovascularization. Retina. 2017;37:687-93.

11. Ikuno Y, Ohno-Matsui K, Wong TY, Korobelnik JF, Vitti R, Li T, et al. Intravitreal aflibercept injection in patients with myopic choroidal neovascularization: The MYRROR Study. Ophthalmology. 2015;122:1220-7.

12. Wolf S, Balciuniene VJ, Laganovska G, Menchini U, OhnoMatsui K, Sharma T, et al. RADIANCE: a randomized controlled study of ranibizumab in patients with choroidal neovascularization secondary to pathologic myopia. Ophthalmology. 2014;121: 682-92.

13. Ohno-Matsui K, Ikuno Y, Lai TYY, Gemmy Cheung CM. Diagnosis and treatment guideline for myopic choroidal neovascularization due to pathologic myopia. Prog Retin Eye Res. 2017;63:92-106.

14. Miura M, Iwasaki T, Goto H. Macular hole formation after intravitreal bevacizumab administration in a patient with myopic choroidal neovascularization. Retin Cases Brief Rep. 2011;5: 149-52.

15. Tufan HA, Gencer B, Kara S. Macular hole after intravitreal bevacizumab injection for choroidal neovascularisation. Clin Exp Optom. 2014;97:178-80.
16. Lai TY, Luk FO, Lee GK, Lam DS. Long-term outcome of intravitreal anti-vascular endothelial growth factor therapy with bevacizumab or ranibizumab as primary treatment for subfoveal myopic choroidal neovascularization. Eye (Lond). 2012;26: 1004-11.

17. Gaucher D, Haouchine B, Tadayoni R, Massin P, Erginay A, Benhamou N, et al. Long-term follow-up of high myopic foveoschisis: natural course and surgical outcome. Am J Ophthalmol. 2007; 143:455-62.

18. Kumagai K, Furukawa M, Ogino N, Larson E. Factors correlated with postoperative visual acuity after vitrectomy and internal limiting membrane peeling for myopic foveoschisis. Retina. 2010;30:874-80.

19. Sun CB, Liu Z, Xue AQ, Yao K. Natural evolution from macular retinoschisis to full-thickness macular hole in highly myopic eyes. Eye (Lond). 2010;24:1787-91.

20. Qi Y, Duan AL, You QS, Jonas JB, Wang N. Posterior scleral reinforcement and vitrectomy for myopic foveoschisis in extreme myopia. Retina. 2015;35:351-7.

21. Ripandelli G, Rossi T, Scarinci F, Scassa C, Parisi V, Stirpe M. Macular vitreoretinal interface abnormalities in highly myopic eyes with posterior staphyloma: 5-year follow-up. Retina. 2012;32:1531-8.

22. Wu PC, Chen YJ, Chen YH, Chen CH, Shin SJ, Tsai CL, et al. Factors associated with foveoschisis and foveal detachment without macular hole in high myopia. Eye (Lond). 2009;23:356-61.

23. Chang JS, Flynn HW Jr, Engelbert M, Shane AR, Smiddy WE, Chang S. Pars plana vitrectomy in patients with myopic macular retinoschisis. Br J Ophthalmol. 2014;98:534-7.

24. Shimada N, Ohno-Matsui K, Baba T, Futagami S, Tokoro T, Mochizuki M. Natural course of macular retinoschisis in highly myopic eyes without macular hole or retinal detachment. Am J Ophthalmol. 2006;142:497-500.

25. Veloso CE, Kanadani TM, Pereira FB, Nehemy MB. Vitreomacular interface after anti-vascular endothelial growth factor injections in neovascular age-related macular degeneration. Ophthalmology. 2015;122:1569-72.

26. Novak MA, Welch RB. Complications of acute symptomatic posterior vitreous detachment. Am J Ophthalmol. 1984;97:308-14.

27. Akiba J. Prevalence of posterior vitreous detachment in high myopia. Ophthalmology. 1993;100:1384-8.

28. Ahn SJ, Park KH, Woo SJ. Subfoveal choroidal thickness changes following anti-vascular endothelial growth factor therapy in myopic choroidal neovascularization. Invest Ophthalmol Vis Sci. 2015;56:5794-5800

29. Sun CB, You YS, Liu Z, Zheng LY, Chen PQ, Yao K, et al. Myopic macular retinoschisis in teenagers: clinical characteristics and spectral domain optical coherence tomography findings. Sci Rep. 2016;6:27952. 